\title{
Integrated modeling of jitter MTF due to random loads
}

\author{
Victor L. Genberg*, Gregory J. Michels \\ Sigmadyne, 803 West Ave, Rochester, NY 14611 \\ *genberg@sigmadyne.com (585)235-7460 \\ Keith B. Doyle** \\ MIT Lincoln Laboratory, Lexington, MA 02420
}

\begin{abstract}
Space borne astronomical telescopes are subjected to random dynamic disturbances from the host spacecraft that create line-of-sight (LoS) jitter errors, which decrease image quality. Special software tools and techniques have been developed to determine the degradation in image quality as measured by the modulation transfer function (MTF) and to identify regions of the telescope to be redesigned in order to minimize the LoS jitter response. A general purpose finite element program is used to find the natural frequencies and mode shapes of the telescope. Each of the optical surfaces for each mode shape is then decomposed into average rigid body motion and elastic deformation. Automated calculation of the LoS equations based on the optical prescription of the telescope provides the LoS response due to expected random loads. The percent contribution of each mode shape to the total LoS jitter is reported that helps pinpoint regions of the telescope structure to redesign. The LoS error due to the random input is then decomposed into drift and jitter components based on a specified sensor integration time. The random jitter is converted to a jitter MTF response function which may be used to modify the MTF function of the nominal optical system yielding the resulting optical system MTF in the operational random environment.
\end{abstract}

Keywords: Integrated analysis, FEA, optomechanics, line-of-sight jitter, MTF, random vibration

\subsection{INTRODUCTION}

Many optical systems, including orbiting telescopes, ground based telescopes, and aircraft cameras are subjected to random dynamic disturbances which cause line-of-sight jitter, degrading optical performance. A useful measure of optical performance is the modulation transfer function (MTF). In a finite element program, the calculation of random response provides PSD response functions for LoS errors and net RMS results. The form of these results is not useful for input to optics codes or for calculation of MTF directly. This paper addresses a software tool that computes MTF directly for random loads applied to finite element models.

\subsection{THEORY}

The analyses described in this section are embedded in the general purpose optomechanical analysis software program SigFit ${ }^{1}$. The most common use of SigFit is to analyze finite element derived optical surface deformations. Deformations are typically fit to polynomials for greater interpretation by the mechanical engineer in the design of an optical mount or formatted into various optical surface types to be represented directly into optical design software for detailed optical performance assessment7,8. Additional SigFit capabilities include the generation of optical performance merit functions using structural response quantities for use in FEA numerical optimization solutions and for use in SigFit's own genetic optimization algorithm. Thermo-optic and stress birefringence effects are assessed using SigFit's bulk optical property algorithms that utilize three-dimensional temperature and stress fields and convert them into optical degradation errors including wavefront and polarization. SigFit also performs mechanical analyses of adaptive optical systems to predict the correctability of systems and optimize adaptive solutions including actuator layouts, actuator type, and the shape of the optical element.

**No MIT Lincoln Laboratory resources or funding were used in furtherance of the findings reported in this paper. 
SigFit offers unique capabilities to perform LoS analyses for optical systems. A ray trace algorithm has been embedded within SigFit to determine the optical sensitivity coefficients for each optical surface and develop the LoS equations. These equations are a powerful means to understand how mechanical loads impact static and dynamic pointing errors of an optical system. The LoS equations generated by SigFit are formatted for general purpose FEA codes and included within the finite element model for normal modes analyses. SigFit utilizes the normal modes to execute harmonic, random, and transient dynamic analyses that enable detailed insight into the behavior and performance of optical systems. The following sections provide an overview of SigFit's unique dynamic analysis capability.

\subsection{Modal analysis and random response}

To calculate random response, FE programs typically go through the following steps:

Calculate natural frequencies and mode shapes.

Calculate the frequency response function (FRF) using modal analysis methods.

Calculate the PSD response function given the PSD input forcing function and FRF.

Generate a RMS response by integrating under the PSD response function.

The calculation of natural frequencies and mode shapes can be thought of as a conversion to principal coordinates of the system. Using this feature, modal analysis, allows the dynamic equations to be diagonalized. The FRF functions can be created from a steady state harmonic response analysis. Using the PSD input forcing function and the FRF of LoS response, the PSD of LoS response as well as pointing errors of each optic can be calculated. The RMS of any response is the square root of the area under the PSD response function shown in Figure 1.

$$
P S D_{\text {resp }}=F R F_{\text {resp }}^{2} P S D_{\text {input }} \quad 1 \sigma_{\text {resp }}=R M S_{\text {resp }}=\sqrt{\text { Area }}
$$

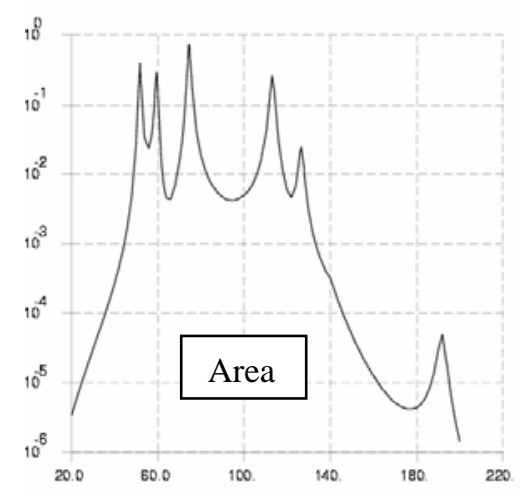

(a) $\mathrm{PSD}_{\text {Resp }}$

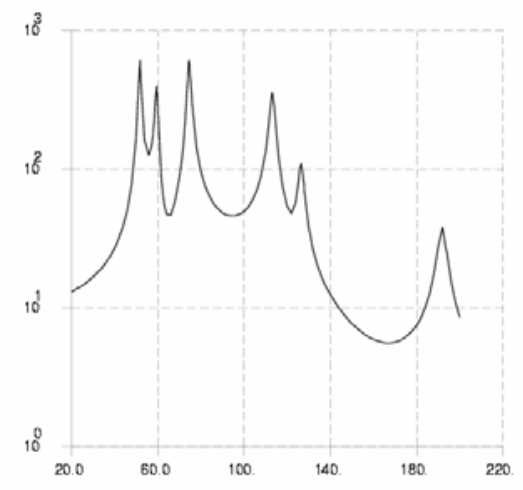

(b) $\mathrm{FRF}_{\text {Resp }}$

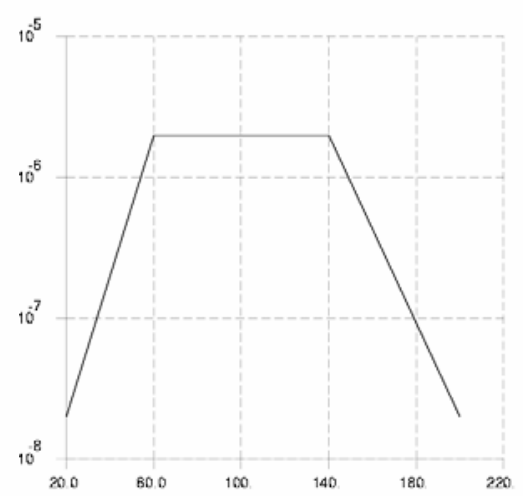

(c) $\mathrm{PSD}_{\text {Input }}$

Figure 1. Random response analysis

If the random analysis is conducted in the FE program, the resulting random response of each node on an optical surface can be calculated. The issue is that each node's value is a positive quantity. The user cannot distinguish between the two dynamic responses shown in Figure xx, since the nodal RMS values are the same. Optically, the two shapes have dramatically different effects of performance. In SigFit, the mode shapes are decomposed into rigid-body motion and elastic distortion. This allows SigFit to distinguish between those two responses in Figure 2 and reports the 1-sigma random response of each surface in rigid-body motions and residual elastic surface RMS. The decomposition of mode shapes is not available in FE programs. 


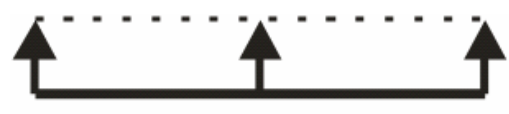

(a)

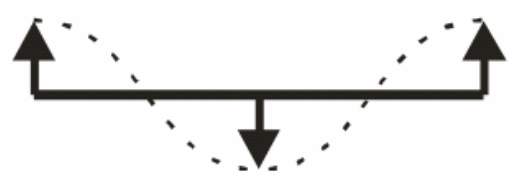

(b)

Figure 2. Deformed surface shapes; (a) all rigid-body motion, (b) all elastic motion

\section{$2.2 \quad$ Line-of-Sight}

Line-of-sight (LoS) errors are calculated in SigFit from a ray trace algorithm. SigFit calculates the LoS coefficients and automatically performs a rigid-body error check to verify their accuracy. LoS results are presented for both image and object spaces as illustrated in Figure 3.

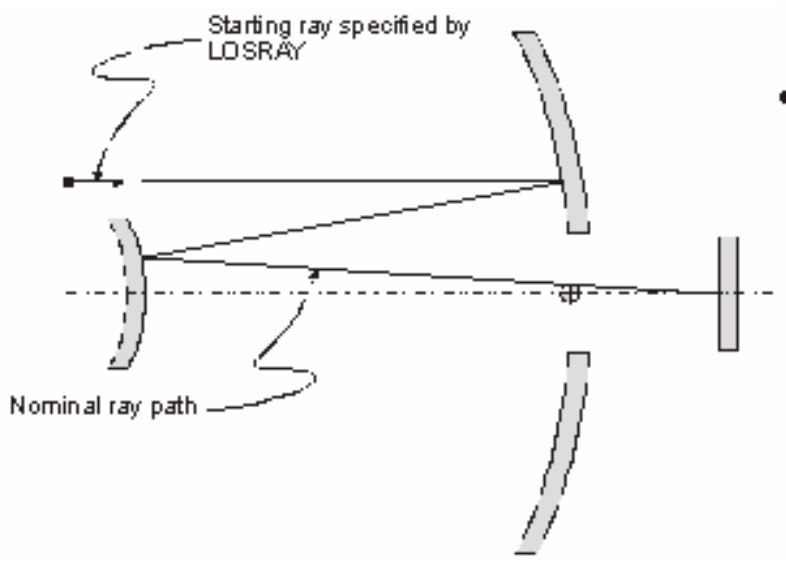

(a)

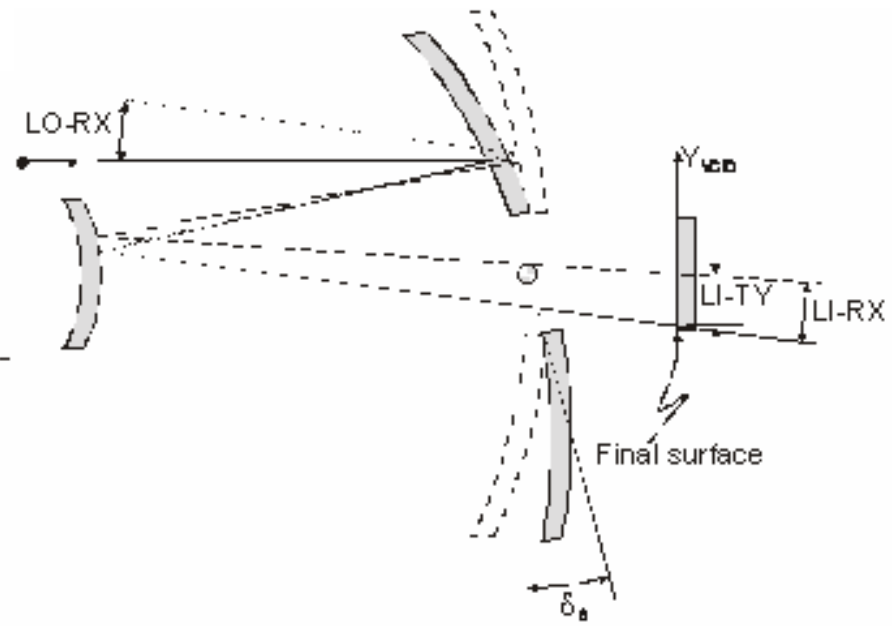

(b)

Figure 3. Line-of-sight in (a) unperturbed system and (b) perturbed system

In harmonic response, the LoS motion has both $\mathrm{X}$ and $\mathrm{Y}$ components (Tx, Ty) which have different phasing (Фx, Фy) at each frequency step due to damping in the structure. At any given frequency step the magnitude of the net $\operatorname{LoS}(\Delta=\mathrm{Tv})$ response can be found from the following equations.

$$
\begin{gathered}
\bar{T}_{x}=T_{x} \cos \left(\Theta+\Phi_{x}\right) \quad \bar{T}_{y}=T_{y} \cos \left(\Theta+\Phi_{y}\right) \\
\Delta^{2}=T_{v}{ }^{2}=T_{x}{ }^{2} \cos ^{2}\left(\Theta+\Phi_{x}\right)+T_{y}{ }^{2} \cos ^{2}\left(\Theta+\Phi_{y}\right) \\
\frac{d T_{v}}{d \Theta}=0 \quad-\tan (2 \Theta)=\frac{T_{x}{ }^{2} \sin \left(2 \Phi_{x}\right)+T_{y}{ }^{2} \sin \left(2 \Phi_{y}\right)}{T_{x}{ }^{2} \cos \left(2 \Phi_{x}\right)+T_{y}{ }^{2} \cos \left(2 \Phi_{y}\right)}
\end{gathered}
$$


The net LoS is used in SigFit's MTF calculation. If the LoS equations are added to the FE model for random response analysis, only the $\mathrm{X}$ and $\mathrm{Y}$ components are available. The vector magnitude is not available.

\subsection{MTF due to jitter}

The approach in SigFit is to use the natural frequencies and mode shapes from the FE program as a starting point. The LoS equations are generated in SigFit, followed by a harmonic response analysis to get the LoS FRF. Using the equations in the above sections, the net LoS RMS response $\left(\Delta_{\mathrm{rms}}\right)$ is calculated. As shown by Lucke ${ }^{4}$, the dynamic response can be divided into two parts, a slow drift (DC) response and a faster jitter (AC) response, where slow and fast are based on the sensor integration time. The weighting factor $\left(W_{d}\right)$ is used to partition the response, where $T$ is the sensor integration time and $f$ is the frequency of the dynamic response in $\mathrm{Hz}$.

$$
\begin{gathered}
\Delta_{r m s}=\sqrt{\int_{0}^{\infty} W_{d}(f) \Delta_{\mathrm{PSD}}(f) d f} \\
W_{d}=1-2(1-\cos (C)) / C^{2} \quad \text { where } C=2 \pi f T
\end{gathered}
$$

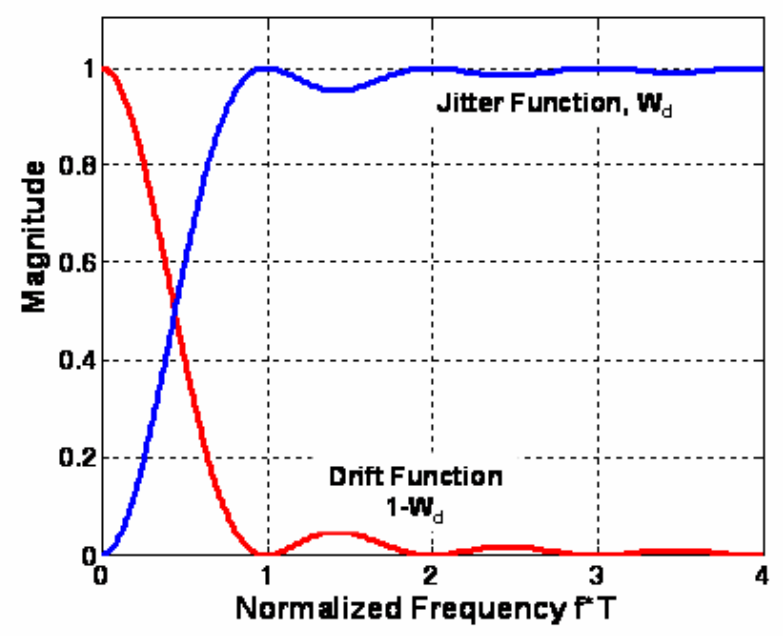

Figure 4. Drift and jitter weighting functions

During SigFit's random response analysis, the jitter MTF effect is calculated from:

$$
\begin{gathered}
M T F_{\text {JitterRandom }}(f)=e^{-2 \pi^{2} \Delta_{r m s}^{2} f^{2}} \\
M T F_{\text {Net }}=M T F_{\text {Nominal }} * M T F_{\text {JitterRandom }}
\end{gathered}
$$




\subsection{CASSEGRAIN TELESCOPE EXAMPLE}

A Cassegrain telescope (Figure 5a) is used as an example with a random base shake input PSD shown in Figure 5b.

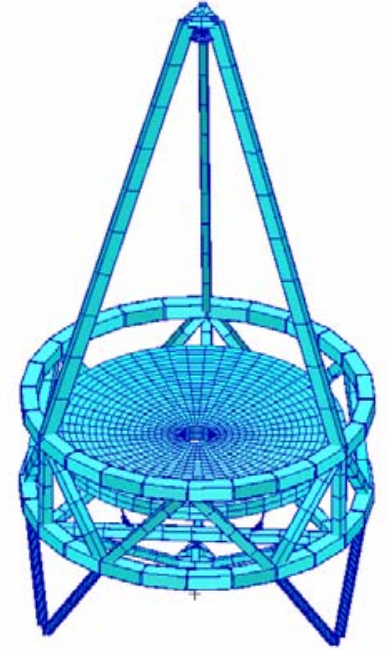

(a)

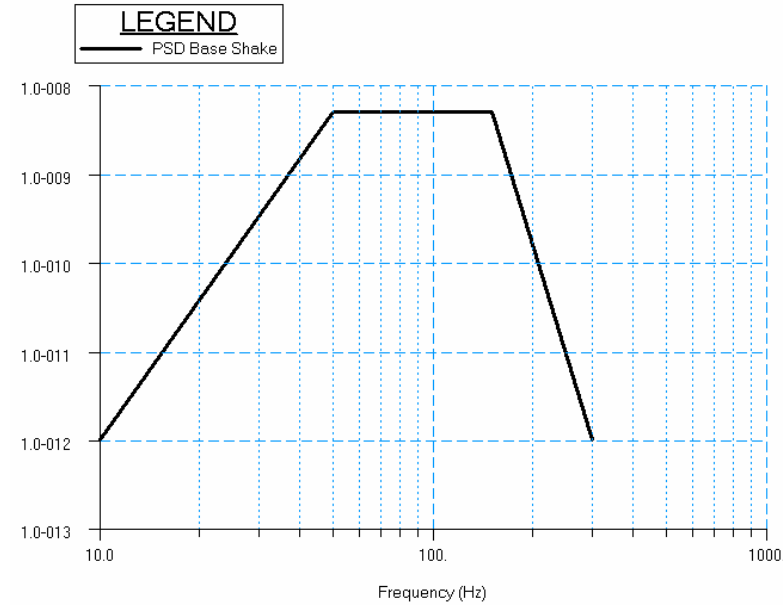

(b)

Figure 5. (a) Simple telescope model and (b) PSD of base shake input

SigFit calculation of LoS coefficients is shown in Table 1, where surface 2 is the primary mirror and surface 3 is the secondary mirror. SigFit automatically calculates a rigid-body error check on LoS equations as shown in Table 2.

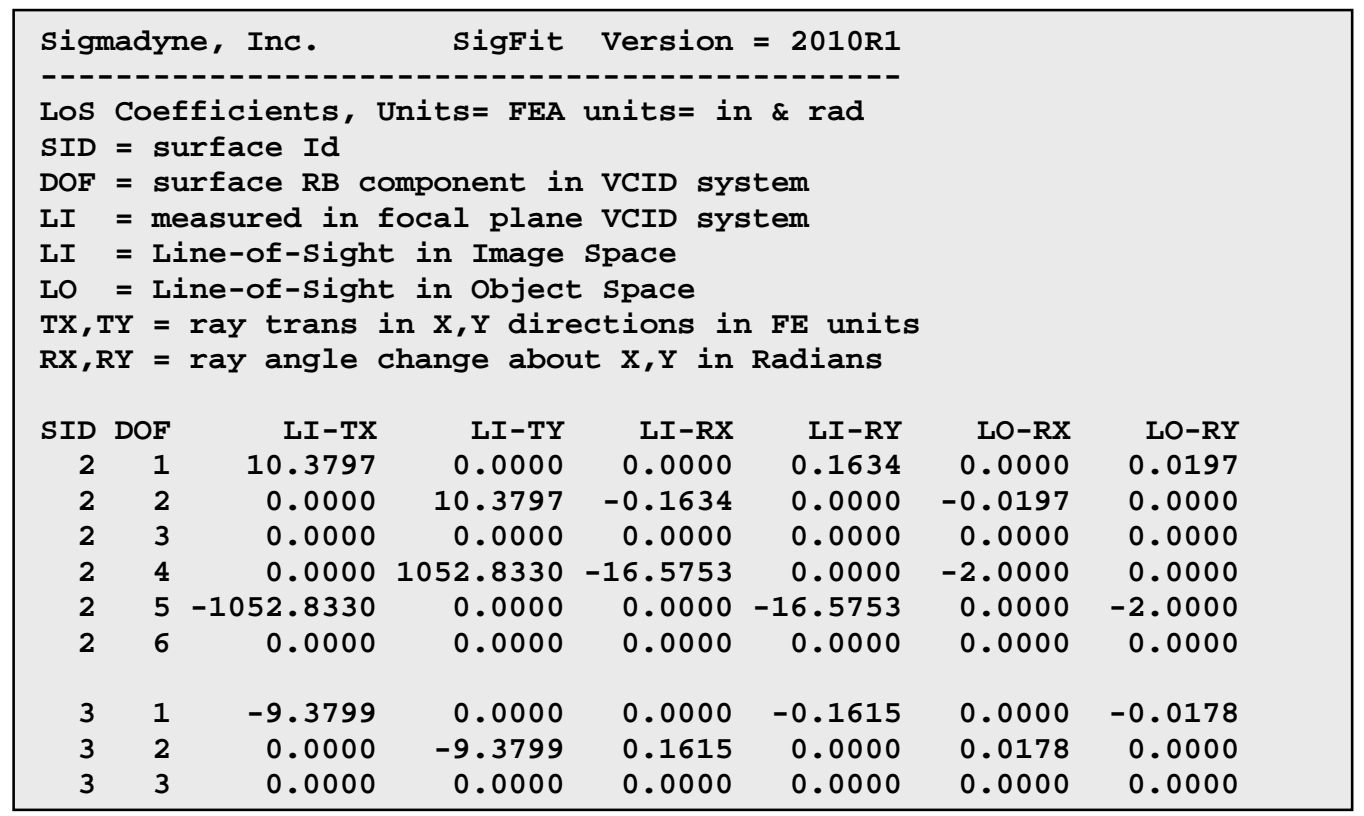

Table 1. Line-of-sight coefficients 


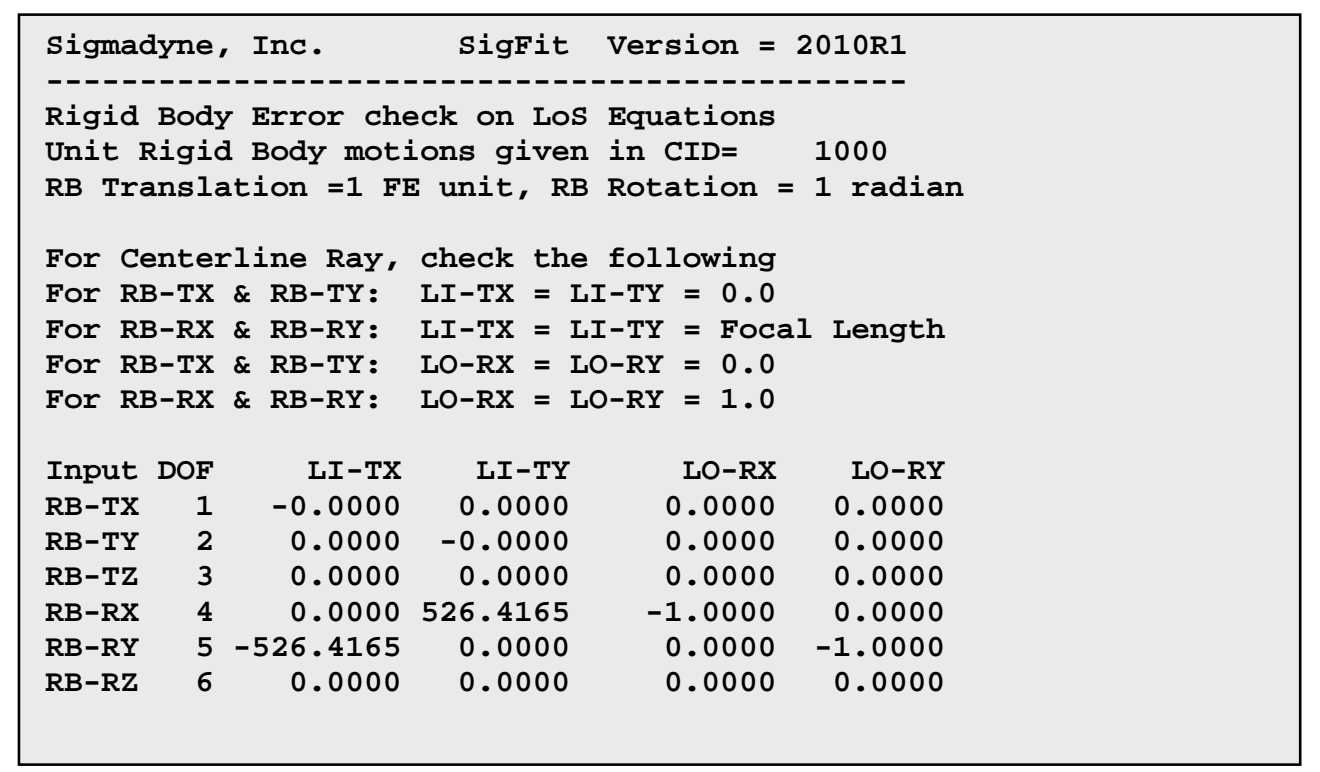

Table 2. Rigid-body error check of the LoS equations

The primary mirror random response summary in Table 3 provides the 1-sigma response of rigid-body motion in six degrees-of-freedom as well as the appropriate vector sums (underlined). The last table entry is the surface RMS after rigid-body motion was subtracted.

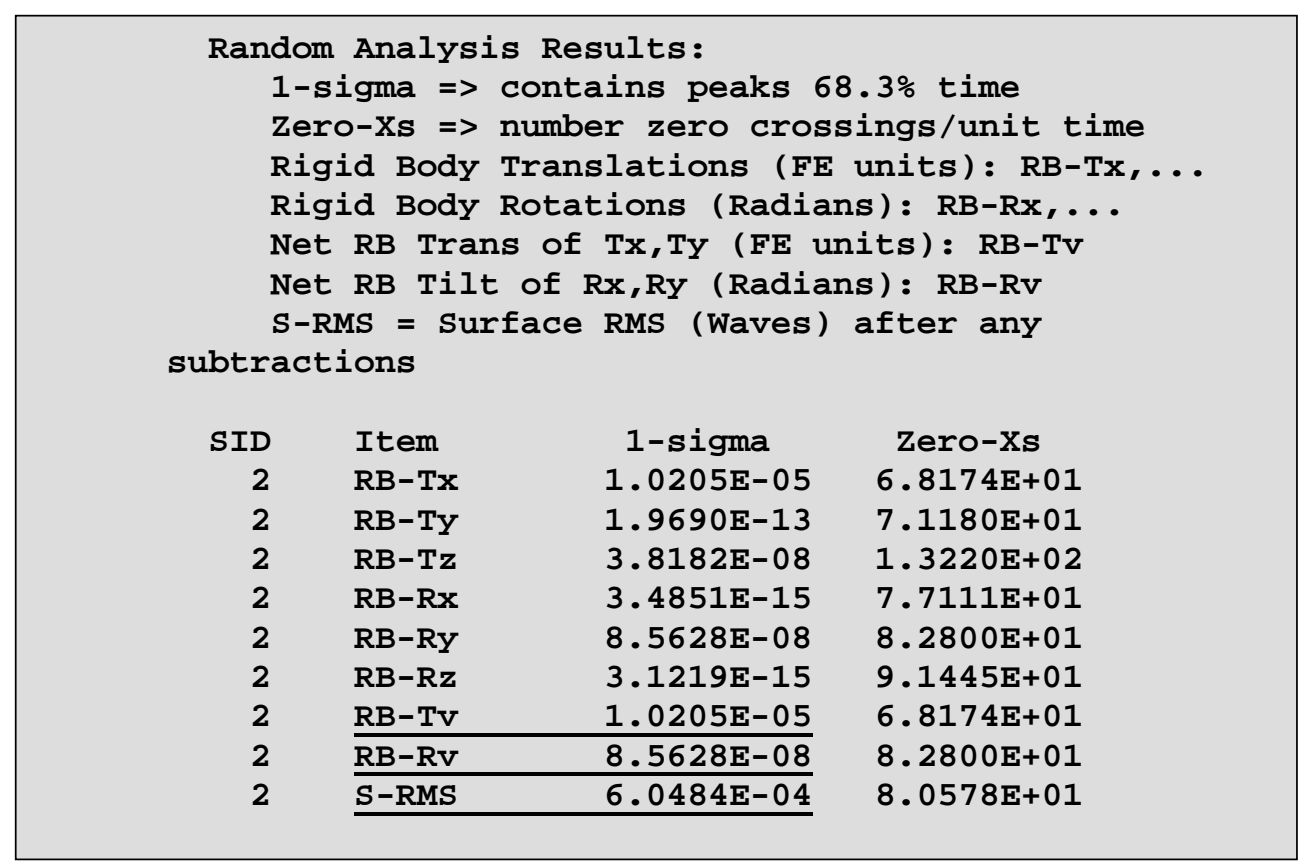

Table 3. Primary mirror random response summary

The impact of sensor integration time on the component and vector sum LoS error is shown in Table 4. 


\begin{tabular}{|c|c|c|c|c|}
\hline \multicolumn{5}{|c|}{ 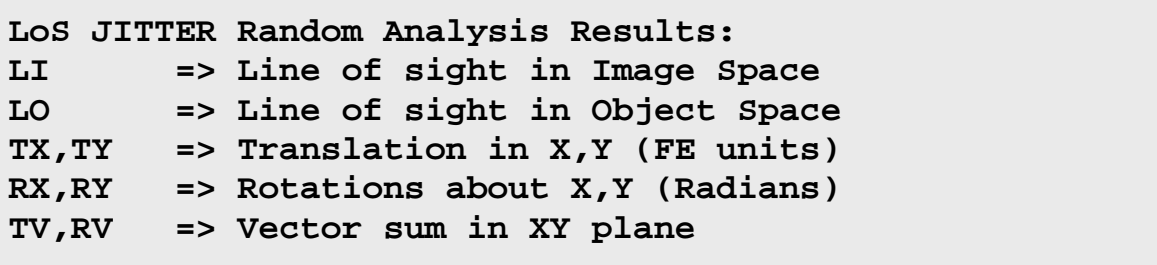 } \\
\hline \multicolumn{5}{|c|}{ Results weighted for integration time $(\mathrm{sec})=0.010000$} \\
\hline & Item & 1-sigma & Zero-Xs & \\
\hline JITTER & LI -TX & 1. 5049E-04 & $7.4131 \mathrm{E}+01$ & \\
\hline JITTER & LI - TY & 4. $0148 E-12$ & $7.7957 \mathrm{E}+01$ & \\
\hline JITTER & LI -TV & 1. 5049E-04 & 7. 4131E+01 & \\
\hline JITTER & LO-RX & $7.6266 \mathrm{E}-15$ & 7. 7957E+01 & \\
\hline JITTER & LO-RY & $2.8588 E-07$ & 7. 4131E+01 & \\
\hline JITTER & LO-RV & $2.8588 E-07$ & $7.4131 \mathrm{E}+01$ & \\
\hline Strehl & Ratio Fac & $(\mathrm{SR}-\mathrm{Fac})=$ & 7.9102E-01 & \\
\hline
\end{tabular}

Table 4. LoS random response summary

The effect of the LoS jitter on the MTF is shown in Figure 6 where the solid curve is the nominal MTF and the long dashed curve is the net MTF after modified by jitter effects.

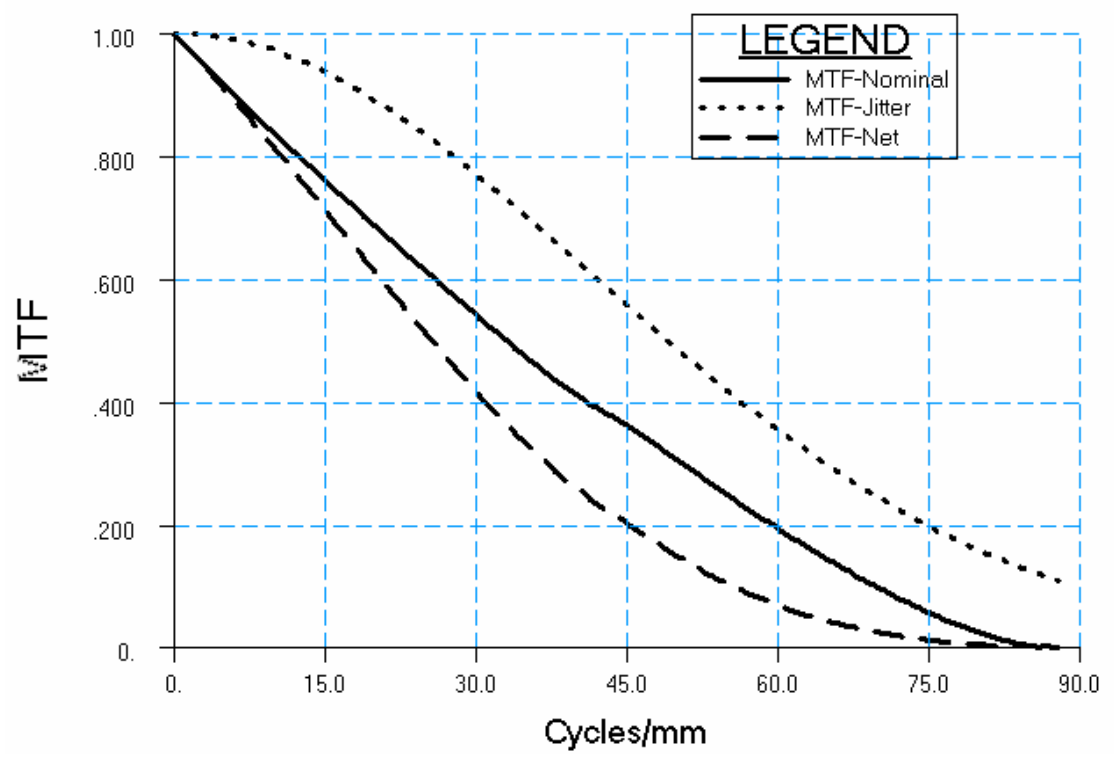

Figure 6. Telescope MTF loss due to jitter

The Strehl ratio factor is computed as the area under the MTF-Net curve divided by the area under the MTF-Nominal curve. The Strehl ratio factor is used to multiply the nominal Strehl ratio of the unperturbed system and provides a single system performance metric in which optimization techniques may be applied. For the telescope example, a Strehl 
ratio factor of 0.791 is computed using a sensor integration time of $0.01 \mathrm{sec}$. As the integration time of the detector becomes smaller, the LoS jitter decreases. Alternatively, if the integration time is infinite then all frequencies contribute to the LoS jitter. In this case, the resulting Strehl ratio factor reduces to 0.766 .

If after the random analysis, the jitter effects on MTF are too large, the design engineer can use the diagnostics calculated by SigFit to find ways to improve the structural design. Table 5 gives each modes contribution to LoS PSD and identifies modes 5 and 7 as key contributors.

\begin{tabular}{|rrrrr|}
\hline \multicolumn{5}{|c|}{ Each modes \% contribution to LoS JITTER PSD } \\
Mode & Freq & LI-TV & LI-RV & \multicolumn{1}{c|}{ LO-RV } \\
4 & 66.15 & 0.000 & 0.000 & 0.000 \\
5 & 66.71 & 70.282 & 81.387 & 70.282 \\
\hline 6 & 76.11 & 0.000 & 0.000 & 0.000 \\
7 & 76.12 & 24.232 & 17.369 & 24.232 \\
\hline 8 & 120.92 & 0.000 & 0.000 & 0.000 \\
9 & 121.39 & 5.432 & 1.113 & 5.432 \\
10 & 122.76 & 0.000 & 0.000 & 0.000 \\
11 & 156.22 & 0.000 & 0.000 & 0.000 \\
12 & 164.24 & 0.000 & 0.000 & 0.000 \\
$\ldots$ & truncated & & & \\
\hline
\end{tabular}

Table 5. Each modes contribution to LoS PSD response

The contribution of each optical surface to the net LoS error is shown in Table 6. For the critical modes 5 and 7, the primary mirror has the largest effect on LoS. The engineer should plot modes 5 and 7 to understand the root cause. Plotting the strain energy density will identify the elements working the hardest. If these elements are increased in size, the jitter effects of modes 5 and 7 will be reduced. In the telescope example, the primary mirror flexures and the main mount strut flexures show the highest strain energy density. A design trade may then be conducted on whether stiffening the flexures to reduce jitter is worth the increase in the loss in mechanical isolation. With such conflicting design requirements, this telescope design is a perfect candidate for design optimization. SigFit's capability to write surface RMS equations in Nastran bulk data form (DRESP2) ${ }^{5}$ and the ability to provide calculated responses such as the Strehl ratio factor (DRESP3) ${ }^{6}$ allow this optimization to be conducted in MSC/Nastran's optimization tool.

\begin{tabular}{|c|c|c|c|c|}
\hline Mode\# & Total-LoS & PM & SM & FP \\
\hline 5 & 1.00 & 0.74 & 0.31 & 0.66 \\
\hline 7 & 1.00 & 1.36 & -0.36 & -0.03 \\
\hline
\end{tabular}

Table 6. Each optical surfaces contribution to a mode’s LoS

\subsection{SUMMARY}

Special software tools and techniques have been developed to determine the degradation in image quality as measured by the modulation transfer function (MTF) and to identify regions of the structure to be redesigned in order to minimize the LoS jitter response. A general purpose finite element program is used to find the natural frequencies and mode shapes of the telescope. Each of the optical surfaces for each mode shape is then decomposed into average rigid body motion and elastic deformation. Automated calculation of the LoS equations based on the optical prescription of the telescope provides the LoS response due to expected random loads. The percent contribution of each mode shape to the total LoS jitter is reported. This identifies regions of the telescope structure to redesign to minimize the response of the telescope. The LoS error due to the random input is then decomposed into drift and jitter components based on a specified sensor integration time. The random jitter is converted to a jitter MTF response function which may be used to modify the MTF function of the nominal optical system yielding the resulting optical system MTF in the operational random environment. 


\section{REFERENCES}

[1] SigFit Reference Manual, Sigmadyne, Inc., Rochester, NY, 2011. (2011)

[2] Doyle, K., Genberg, V., Michels, G., Integrated Optomechanical Analysis, SPIE, TT58, (2002)

[3] Doyle, K.B., Line-of-sight jitter analysis for MLCD, New Developments in Optomechanics, SPIE Vol. 6665(17), August, 2007.

[4] Lucke, R. L., Sirlin, S. W., San Martin, A. M., New Definitions of Pointing Stability: AC and DC Effects, The J of Astronautical Sciences, Vol. 40, No.4, Oct-Dec 1992, pp 557-576.

[5] Genberg, V., “Optical Performance Criteria in Optimum Structural Design”, SPIE Paper 3786-29, July 1999.

[6] Michels, G., Genberg, V., Doyle, K., Bisson, G., " Design optimization of system level adaptive optical performance", SPIE Paper 5867-25, 2005

[7] Genberg, V., Doyle, K. and Michels, G., “An optical interface for MSC Nastran,” MSC Conference Proc VPD04-31, (2004).

[8] Michels, G., Genberg, V., and Doyle, K., “Integrating ANSYS mechanical analysis with optical performance analysis with SigFit,” ANSYS User Conference Proc., (2008).

[9] Michels, G., Genberg, V., Doyle, K., and Bisson, G., "Design optimization of actuator layouts of adaptive optics using a genetic algorithm,” Proc. SPIE 5877-22, (2005). 